\title{
IGFBP7 aggravates sepsis-induced acute lung injury by activating the ERK1/2 pathway
}

\author{
Qiaolian Xu' ${ }^{1}$, Jun Wang ${ }^{2}$ \\ ${ }^{1}$ Department of ICU, Nanjing First Hospital, Nanjing Medical University, Nanjing, Jiangsu Province, \\ 210009, China \\ ${ }^{2}$ Department of Critical Care Medicine, Tongde Hospital of Zhejiang Province, Hangzhou, Zhejiang \\ Province, 310012, China
}

\begin{abstract}
Introduction. Sepsis is characterized by an infection-caused acute inflammatory response, which is usually accompanied by multiple organ failure, especially lung injury. During sepsis, a large number of endotoxins such as lipopolysaccharides (LPSs) are secreted from Gram-negative bacteria. However, the mechanisms underlying acute lung dysfunction caused by sepsis have not yet been well defined.

Material and methods. To identify the mechanism of insulin-like growth factor binding protein 7 (IGFBP7) in acute lung injury during sepsis, the effects of IGFBP7 shRNA were evaluated in a model of cecal ligation puncture (CLP)-induced sepsis in mice. Histologic evaluation of the effects of IGFBP7 on CLP-induced acute lung injury was performed by H\&E staining. Murine pulmonary microvascular endothelial cells (MPVECs) were transfected with shIGFBP7 or shNC before treatment with LPS to mimic the sepsis-induced lung dysfunction. The effects of CLP or LPS on IGFBP7 expression and the activation of ERK1/2 pathway were analyzed by western blot. MTT and LDH assays were used to measure the viability of MPVECs under different treatment regimes. The apoptosis rate of MPVECs in different groups was detected by flow-cytometry analysis.

Results. IGFBP7 was strongly up-regulated in sepsis-induced acute lung injury in mice. IGFBP7 silencing attenuated sepsis-induced apoptosis and cytotoxicity in MPVECs. Furthermore, the activation of ERK1/2 pathway was regulated by IGFBP7 during sepsis-induced inflammation. IGFBP7 inhibition by RNA interference in MPVECs attenuated CLP-induced morphological features of lung dysfunction. The knockdown of IGFBP7 attenuated LPS-induced MPVECs' apoptosis by the suppression of the ERK1/2 pathway.

Conclusions. We demonstrated for the first time that IGFBP7 is involved in the pathogenesis of sepsis-induced acute lung injury and may serve as a therapeutic target in sepsis-induced acute lung injury. (Folia Histochemica et Cytobiologica 2020, Vol. 58, No. 4, 247-254)
\end{abstract}

Key words: mouse; CLP sepsis; acute lung injury; MPVECs; IGFBP7; ERK1/2; siRNA; apoptosis

\section{Introduction}

Sepsis is characterized by an infection-caused acute inflammatory response to tissue injury and is often caused by bacterial, fungal, and viral infections [1-3]. Sepsis is a clinical disorder with a high mortality rate

Correspondence address: Jun Wang,

Department of Critical Care Medicine,

Tongde Hospital of Zhejiang Province,

No. 234 Gucui Road, Xihu District,

Hangzhou City, Zhejiang Province, China

phone: $86+0571-88695621$

e-mail: wangjun202008@126.com and is companied with numerous health problems worldwide $[4,5]$. If the inflammatory response is particularly severe, the homeostasis of multiple organ system would be disrupted [6]. Severe sepsis is usually accompanied by multiple organ failure, and in the process of organ dysfunction, the lung is frequently the first to fail $[7,8]$. The pathogenesis of sepsis-induced acute lung injury was related to the overexpression of cytokine-mediated inflammation [9]. Within sepsis, a large number of endotoxins such as lipopolysaccharides (LPSs) are commonly secreted from Gram-negative bacteria [10]. LPS activates Toll-like-receptor-4 and co-receptor CD14, thereby 
triggering the mitogen-activated protein kinase signaling pathways, including the p38, extracellular signal-related kinase pathway (ERK), and c-Jun $\mathrm{N}$-terminal kinase pathways $[11,12]$. However, the mechanisms underlying acute lung dysfunction caused by sepsis have not yet been well defined.

Insulin-like growth factor (IGF) signaling contributes to cell growth and differentiation and continues its role throughout life by triggering cell proliferation and inhibiting cell apoptosis [13]. Some studies have indicated that IGF-binding proteins (IGFBPs) acts as a part of transporters of IGFs, extending their half-life, and regulating their access to their receptors $[14,15]$. IGFBP7 is a secreted protein with IGF-1, insulin and activin A binding properties that possess IGF-independent activity [16, 17]. IGFBP7 is a potential tumor endothelial cell marker that is expressed at higher levels in tumor-associated endothelium than in normal endothelial cells. Tumorigenesis [18], the cellular senescence and apoptosis are also regulated by IGFBP7 through the ERK signaling pathway [19-21]. Recently, multiple studies have revealed that the ERK pathway plays an important role in the pathogenesis of LPS-induced lung injury [22, 23]. However, the effect of IGFBP7 in sepsis-induced acute lung injury remains unclear. In the present study, we designed and performed experiments to demonstrate the potential mechanism of IGFBP7 and ERK signaling pathway in sepsis-induced acute lung injury in MPVECs and mice. In this study we provide evidence that knockdown of IGFBP7 suppressed sepsis-induced acute lung injury through ERK1/2 signaling inhibition.

\section{Methods}

Animal model. Wild type C57BL/6J male mice (8-10 weeks of age, weight $18 \pm 2 \mathrm{~g}$ ) were obtained from Weitong Lihua Biology Company (Beijing, China). All animal experiments were approved by the Experimental Animal Welfare Ethics Committee of Zhejiang Academy of Traditional Chinese Medicine and conducted in accordance with the guidelines of the Animal Care. Twenty-four mice were randomly divided into four experimental groups: (1) sham group, (2) cecal ligation puncture (CLP) group, (3) CLP + Ad-shNC group, and (4) CLP + Ad-shIGFBP7 group. Before the surgery, all animals were anesthetized by intraperitoneal administration of pentobarbital $(50 \mathrm{mg} / \mathrm{kg})$. The sham group without ligating or puncturing the cecum served as sham-operated. The CLP group was subjected to cecal ligation and puncture (CLP) surgery [24]. The animals received injection of negative control (NC) Ad-shNC or Ad-shIGFBP7 (5' GGACAUCUGGAACGUCACUTT 3') before the CLP surgery. To arise the knockdown efficiency, a second administration of the adenovirus was performed 72 hours later. Two weeks after the first injection, the mice were ready for use in the experiments. One week later, mice were anesthetized with pentobarbital sodium and both lungs were harvested and kept frozen at $-80^{\circ} \mathrm{C}$ until analysis.

Hematoxylin and eosin (H\&E) staining. The lung tissues from the mice were fixed with $10 \%$ buffered paraformaldehyde for 24 hours, then embedded in paraffin and sectioned at a thickness of $5 \mu \mathrm{m}$. After hematoxylin and eosin (H\&E) staining, the pathological changes of lung tissues were graded using the double-blind method.

Cell culture. The MPVECs were isolated from C57BL/6 mice. Shortly, mice were anesthetized with pentobarbital sodium and lungs were isolated from the thoracic cavity and perfused with phosphate-buffered saline (PBS) containing heparin. The lateral lobe was cut into small pieces under sterile conditions, placed in a dish which coated with $0.1 \%$ gelatin and cultured in DMEM (Gibco, Invitrogen, USA) with $20 \%$ FBS. The expression of cluster of differentiation 31 (CD31) was used for the identification of the characterization of primary cultured MPVECs [25]. Cells were transfected with shIGFBP7 or shNC lentiviral particles with $10 \mu \mathrm{g} / \mathrm{ml}$ Polybrene (Santa Cruz Biotechnology Inc., Santa Cruz, CA, USA) before co-treatment with LPS for 48 hours. After incubation with lentiviral particles for $24 \mathrm{~h}$, transduced cells were selected with puromycin (Sigma-Aldrich, Milwaukee, WI, USA).

Western blot analysis. The total protein was extracted from the lung tissue of C57BL/6 mice and MPVECs. The protein concentrations in the lung tissue homogenate or lysate of MPVECs were detected by BCA Protein Assay Kit (23227, Thermo Fisher Scientific, San Jose, CA, USA). The protein samples were separated on $10 \%$ SDS-Polyacrylamide gel and transferred onto nitrocellulose membrane. After blocking with 5\% nonfat milk, the membrane was incubated with specific primary antibodies purchased from Abcam (Abcam, Cambridge, MA, USA), including IGFBP7 (ab74169), ERK1/2 (ab17942), p-ERK1/2 (ab214362) and actin (ab179467), Abcam) at $4^{\circ} \mathrm{C}$ overnight, followed with incubation with secondary antibodies (ab131368, Abcam) at room temperature. The signals were measured by Image J software (US National Institutes of Health, Bethesda, MD, USA).

Cell viability assay. The viability of MPVECs was assessed by the MTT assay kit (ab211091, Abcam) according to the manufacturer's instructions. Cells were seeded into 96-well plates at a density of $5 \times 10^{4} /$ well. After experiment, MTT solution was added into each well and incubated at 37 for 5 hours. Then, the medium was removed and DMSO was added into each well. The absorbance of the developed color 


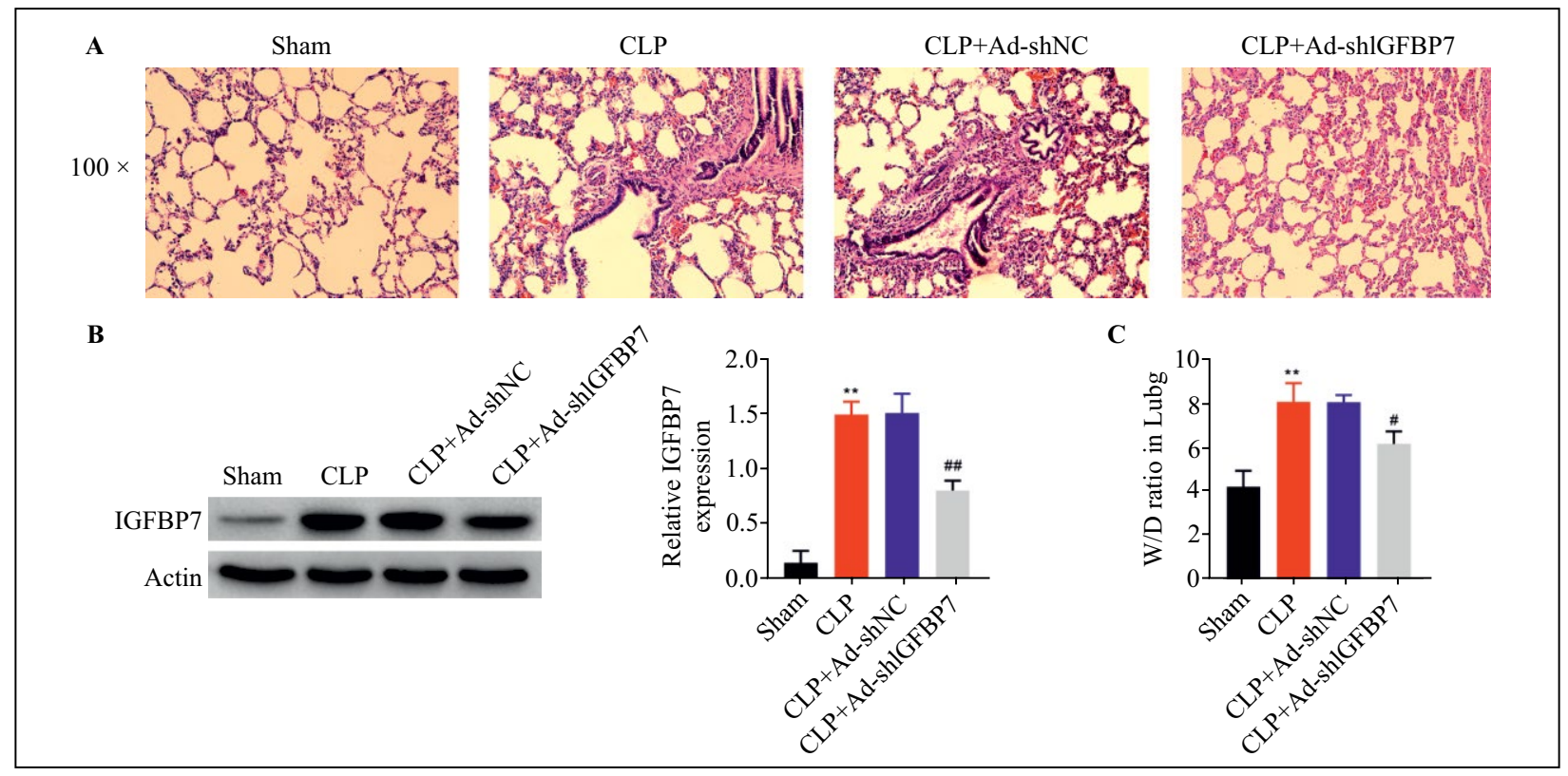

Figure 1. Effects of IGFBP7 in cecal ligation and puncture-induced acute lung injury. A. The lung tissue sections were staining by hematoxylin and eosin. Representative morphological changes of the lung tissues obtained from mice of different groups. B. The expression levels of IGFBP7 were assessed by western blot. C. The lung W/D (wet-to-dry) ratio from different groups. ** $\mathrm{p}<0.001$ compared with Sham; ${ }^{\# \#} \mathrm{p}<0.001$ compared with CLP.

$(590 \mathrm{~nm})$ was detected by a microplate reader (Bio-Rad, Hercules, CA, USA).

Lactate Dehydrogenase (LDH) release assay. To determine the activity of cytoplasmic enzyme released by damaged MPVECs, LDH release was measured by LDH kit (ab65393, Abcam) according to the manufacturer's instructions. The intensity of released LDH in the culture was measured at $490 \mathrm{~nm}$ in a microplate reader. The cell viability was reflected by the rate of $\mathrm{LDH}$ activity released into the medium to total cellular LDH activity.

Cell apoptosis analysis. After being transfected with or without shIGFBP7 and treated with LPS, MPVECs were harvested and the apoptosis rate was analyzed by Annexin V-fluorescein isothiocyanate (FITC) apoptosis detection kit (ab14085, Abcam) according to the manufacturer's instruction. Cells were resuspended to $0.5 \times 10^{6}$ cells $/ \mathrm{mL}$ in Annexin $\mathrm{V}$ binding buffer containing propidium iodide (PI) and Annexin V-FITC, and incubated for $15 \mathrm{~min}$ at room temperature in the dark. Cell apoptosis in MPVECs was measured with flow cytometry (BD Biosciences, San Diego, CA, USA) using the $488 \mathrm{~nm}$ detector for Annexin V-PI.

Statistical analysis. Biochemical values are expressed as mean \pm standard deviation (SD). Statistical differences for multiple groups and the significance were evaluated using one-way analysis of variance followed by Student Newman-Keuls test. $P$ values less than 0.05 were considered significant.

\section{Results}

\section{Knockdown of IGFBP7 attenuated cecal ligation and puncture-induced acute lung injury in mice}

To identify the mechanism of IGFBP7 action in acute lung injury during sepsis, the protective effect of IGFBP7 shRNA was evaluated in CLP-induced sepsis in mice. Male C57BL/6 mice were randomly divided into four groups: sham, CLP model, CLP + shNC, and CLP + shIGFBP7. Two weeks after the first shIGFBP7 or shNC injection, the mice were used for the experiments.

The effects of IGFBP7 on CLP-induced acute lung injury were histologically evaluated. Compared with the sham group, the lung tissues isolated from the CLP and CLP + shNC groups showed abnormal histological features, including acute alveolar damage, congestion, and thickened alveolar walls (Fig. 1A). However, injected with IGFBP7 shRNA after CLP modeling significantly prevented CLP-induced acute lung-tissue damage.

The effect of CLP on IGFBP7 expression was analyzed after CLP modeling by western blot. Compared with the sham group, the expression levels of IGFBP7 in lung tissue were significantly increased after CLP modeling. IGFBP7 shRNA treatment reduced IGFBP7 expression compared with that of the CLP group (Fig. 1B). The lung wet-to-dry (W/D) weight ratio also significantly increased after CLP-induced sepsis while significantly decreased in the CLP + 
A

B

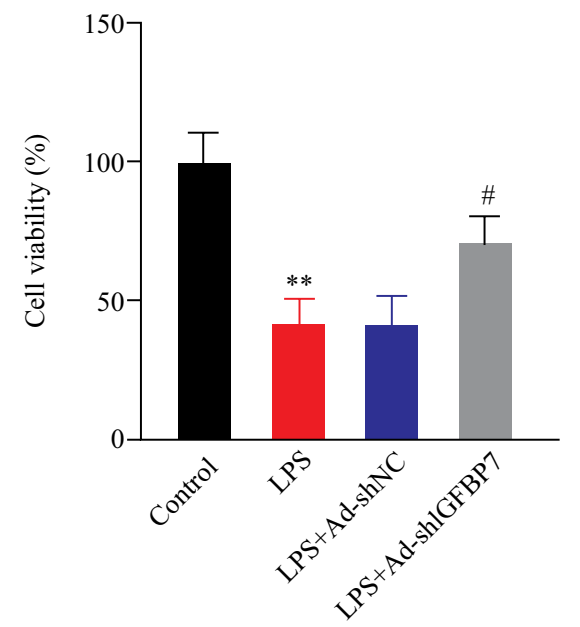

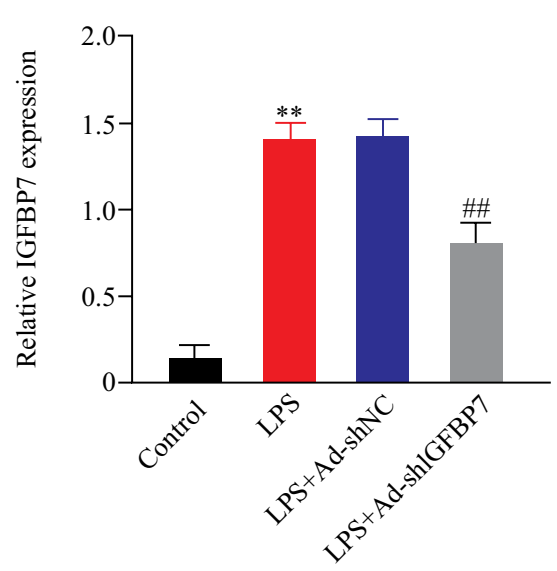

C

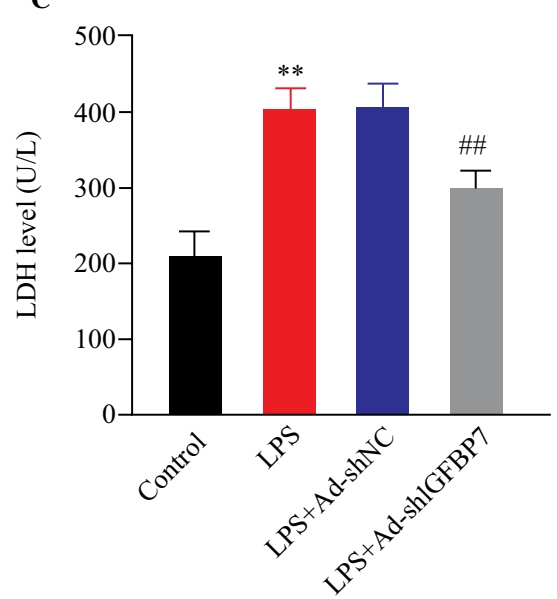

Figure 2. IGFBP7 knockdown attenuates LPS-induced acute injury in MPVECs. MPVECs were transfected with shIGF-BP7 or shNC and treated with or without LPS $(2 \mu \mathrm{g} / \mathrm{mL})$ for $48 \mathrm{~h}$. A. IGFBP7 expression was measured by western blot. B. MPVECs were treated with LPS and cell viability was measured by MTT assay. C. The cytotoxicity level in different groups was measured by the LDH assay. ${ }^{* *} \mathrm{p}<0.001$ compared with the control group; ${ }^{\# \#} \mathrm{p}<0.001$ compared with LPS group.

shIGFBP7 group (Fig. 1C). These results suggested that inhibition of IGFBP7 attenuated CLP-induced lung changes and thus may be critical in regulating lung function during sepsis.

\section{Knockdown of IGFBP7 attenuated LPS-induced acute damage of MPVECs}

To investigate the role of IGFBP7 in sepsis-induced acute lung injury, LPS induction of MPVECs was used to mimic the sepsis-induced lung dysfunction. MPVECs were transfected with shIGFBP7 or shNC before treatment with LPS $(5 \mu \mathrm{g} / \mathrm{mL})$ for $48 \mathrm{~h}$ (the dose of LPS and duration of treatment were based on previous study [26]). LPS treatment was found to significantly up-regulate the levels of IGFBP7 compared with the control group (Fig. 2A). Conversely, shIGFBP7 transfection significantly decreased the LPS-induced IGFBP7 expression compared with MPVECs without transfection.
MTT assay showed that the viability of MPVECs in the LPS treatment group was significantly lower than in the control group, but not in the group transfected with shIGFBP7 (Fig. 2B). To explore the protective mechanism of IGFBP7 inhibition on LPS-induced cellular injury, cytotoxicity was measured by LDH assay. As expected, LPS treatment significantly induced MPVECs' damage by increasing LDH release, whereas the shGFBP7 transfection significantly attenuated the LPS-induced LDH release from MPVECs (Fig. 2C). These results indicated that knockdown of IGFBP7 attenuated LPS-induced negative changes in MPVECs.

\section{Knockdown of IGFBP7 suppressed LPS-induced apoptosis of MPVECS}

To investigate the involvement of IGFBP7 in the regulation of LPS-induced apoptosis, the apoptosis rate in different groups was determined by flow-cytometry 


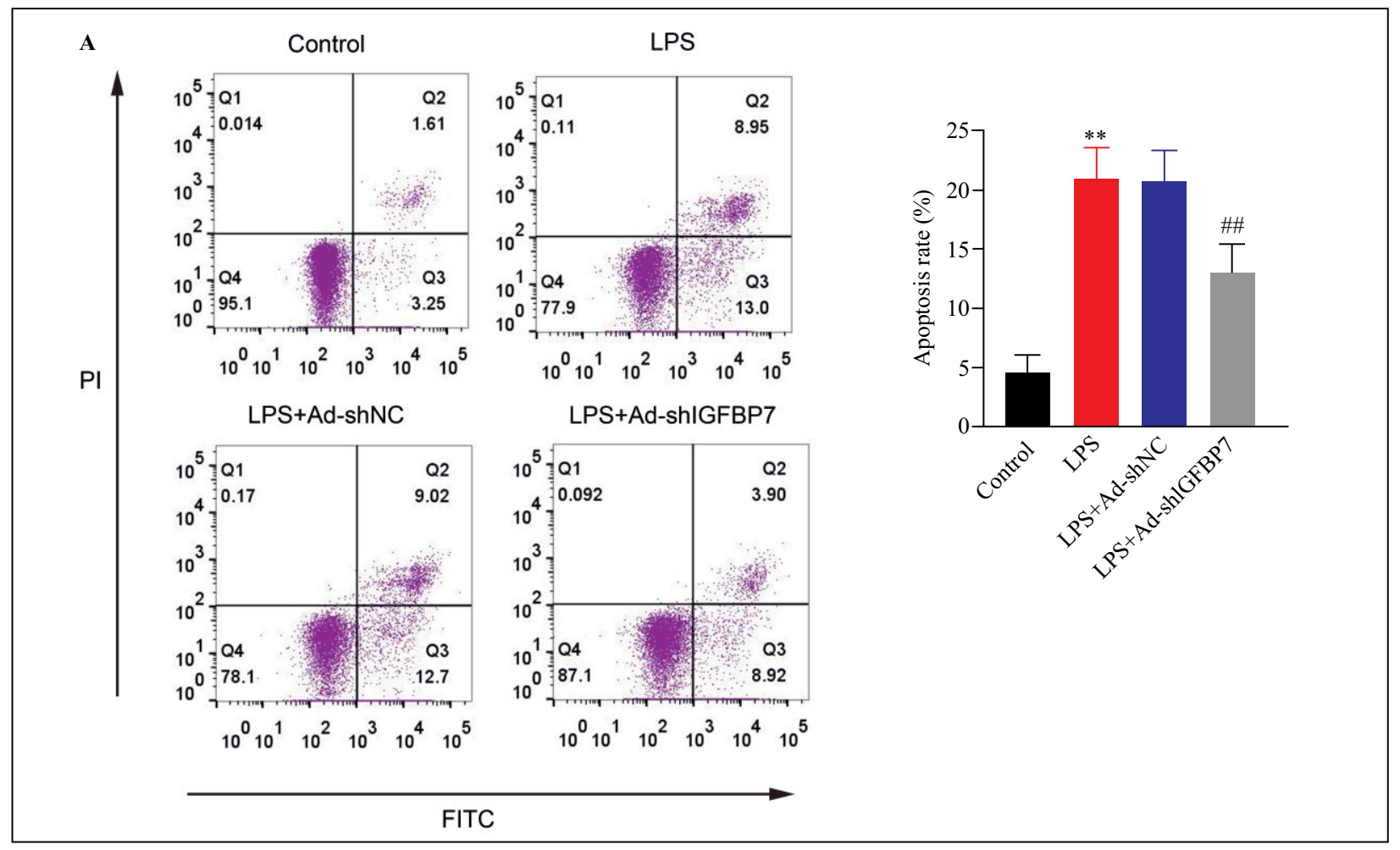

Figure 3. IGFBP7 knockdown suppresses LPS-induced cellular apoptosis in MPVECs. MPVECs were transfected with shIGFBP7 or shNC and treated with or without LPS $(2 \mu \mathrm{g} / \mathrm{mL})$ for $48 \mathrm{~h}$. The apoptosis rate in different groups was measured by flow cytometry. ${ }^{*} \mathrm{p}<0.001$ compared with the control group; ${ }^{\# \#} \mathrm{p}<0.001$ compared with LPS group.

analysis. As shown in Figure 3, the apoptosis rate of MPVECs was significantly higher in the LPS-treated cells than in the control group. Furthermore, when shIGFBP7 treatment was conducted in the LPS-induced MPVECs the cellular apoptosis rate was significantly suppressed. These results indicated that the knockdown of IGFBP7, contributed to the reduction of LPS-induced apoptosis of MPVECs.

\section{Knockdown of IGFBP7 inhibited the LPS-induced activation of the ERK1/2 pathway in MPVECs}

To clarify the potential mechanism by which IGFBP7 regulated LPS-induced MPVECs' damage, activation of ERK1/2 pathway was measured by western blot. LPS induction significantly enhanced the expression of $p$-ERK1/2 but not that of total ERK1/2. By contrast, treatment of LPS-treated cells with shIGFBP7 significantly decreased the expression levels of p-ERK1/2 in MPVECs (Fig. 4). Therefore, knockdown of IGFBP7 may alleviate the LPS-induced activation of the ERK1/2 pathway in MPVECs.

\section{Discussion}

Sepsis is a common and serious condition in surgical patients. It can be caused by a serious bacterial infection in the abdominal cavity and is accompanied by fever with or without hypotension [27]. A critical characteristics of abdominal sepsis is the release of endotoxins inducing the overexpression of the innate immune system and inflammatory responses that lead to tissue injury in the lungs and other organs $[28,29]$. Acute lung injury is the primary complication in sepsis during the continuous development of multiple organ dysfunction [30, 31]. Lung edema, inflammatory cell infiltration, and intrapulmonary hemorrhage have been identified as the typical pathological manifestations of sepsis-induced acute lung injury [32]. The change of the alveolar capillary membrane permeability, inflammatory cell aggregation by production of inflammatory chemokines and release of other inflammatory mediators have been identified as characteristics of acute lung injury.

In the present study, we examined the essential mechanisms of IGFBP7 influence on CLP-induced acute lung injury in mice. Our findings showed that the expression level of IGFBP7 in lung tissue was significantly increased after CLP modeling, and knockdown of IGFBP7 suppressed pro-inflammatory response in lung tissue, such as alveolar-wall thickening, and acute alveolar damage. In addition, IGFBP7 silencing in MPVECs markedly suppressed cell apoptosis and ERK1/2 activation in LPS-stimulated cells. These 


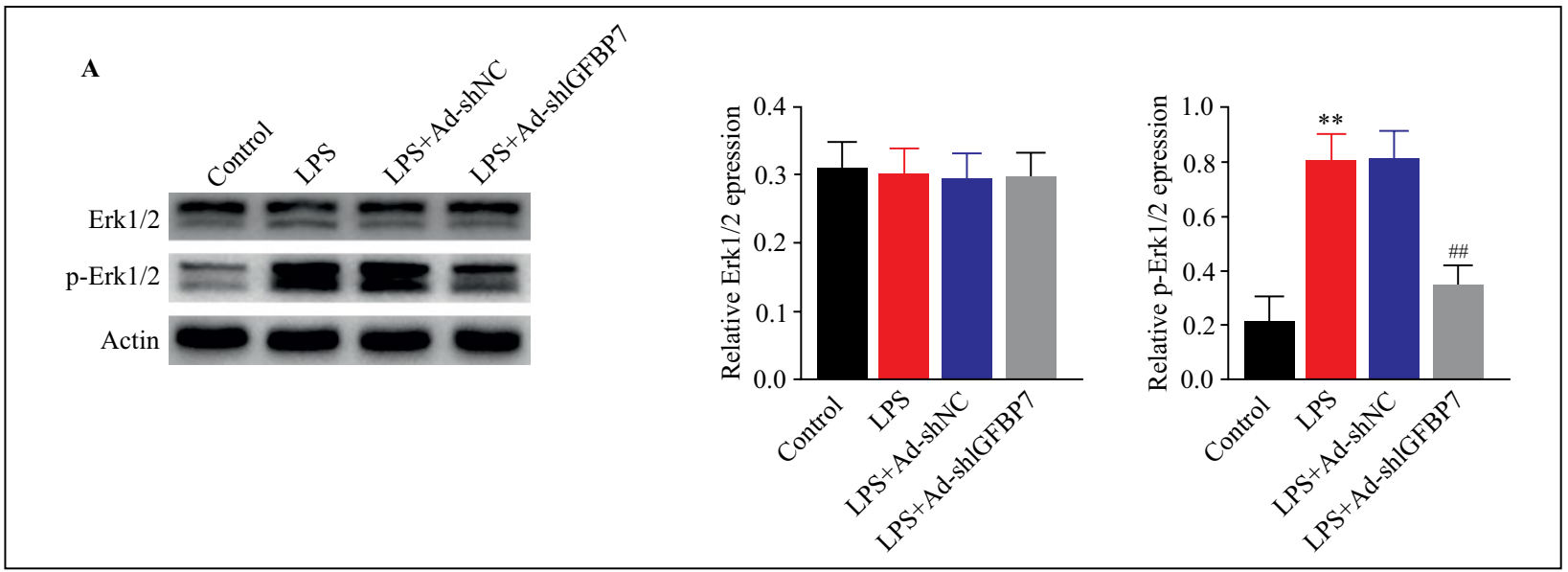

Figure 4. Effects of IGFBP7 on LPS-induced ERK1/2 activation in MPVECs. MPVECs were transfected with shIGFBP7 or shNC and treated with or without LPS $(2 \mu \mathrm{g} / \mathrm{mL})$ for $48 \mathrm{~h}$. The expression of ERK1/2 and p-ERK1/2 was measured by western blot. ${ }^{* *} \mathrm{p}<0.001$ compared with the control group; ${ }^{\# \#} \mathrm{p}<0.001$ compared with LPS group.

changes in lung-tissue pathophysiology were related to the altered viability of MPVECs.

Several molecules are involved in the pathogenesis of sepsis-induced injury, which is usually accompanied by the robust activation of complement system. The pathogen is removed; however, inflammation and organ damage still ensue. Extensive evidence suggests that IGFBP7 could regulate cell proliferation, adhesion, senescence, migration, apoptosis, and angiogenesis in breast, lung, and colorectal cancers [16]. Previous studies have reported that IGFBP7 induces cell-cycle arrest at G1 phase by directly enhancing the expression of p21 and p53, which could function as a biomarker of sepsis-induced acute kidney injury [ 26 , 33]. In the present study, the expression levels of IGFBP7 in lung tissue were significantly increased after sepsis-induced acute lung injury, and the inhibition of IGFBP7 attenuated sepsis-induced lung dysfunction. These results indicated that IGFBP7 was involved in sepsis-induced lung tissue dysfunction. Vizioli et al. reported that IGFBP7 expression markedly enhances the activation of the mitogen-activated protein kinase ERK1/2, and ultimately results in the secretion of pro-inflammatory cytokines [34]. The ERK1/2 pathway also plays a critical role in IL-13-induced lung inflammatory response and LPS-induced acute lung injury [35]. Similar results were obtained in the present study, i.e., knockdown of IGFBP7 alleviated the LPS-induced activation of the ERK1/2 pathway in MPVECs. Other investigators received similar results regarding IGFBP7-dependent ERK1/2 activation in sepsis-induced acute kidney injury [26]. This finding corroborated our results regarding sepsis-induced acute lung injury and indicated that the IGFBP7 regulation of lung-tissue damage was ERK dependent.
In conclusion, this research demonstrated for the first time that IGFBP7 was strongly up-regulated in sepsis-induced acute lung injury, thereby aggravating the lung inflammatory response and organ dysfunction. IGFBP7 silencing attenuated sepsis-induced apoptosis and cytotoxicity in MPVECs. Furthermore, the activation of ERK1/2 was regulated by IGFBP7 through sepsis-induced inflammation. Overall, IGFBP7 may serve as a diagnostic marker and therapeutic target in sepsis-induced acute lung injury; however, further molecular and clinical studies are still needed.

\section{Acknowledgements}

Not applicable.

\section{Funding}

Not applicable.

\section{Competing interests}

The authors state that there are no conflicts of interest to disclose.

\section{Ethics approval}

Ethical approval was obtained from the Experimental Animal Welfare Ethics Committee of Zhejiang Academy of Traditional Chinese Medicine.

\section{Statement of Informed Consent}

Not applicable. 


\section{Availability of data and materials}

All data generated or analyzed during this study are included in this published article.

\section{Authors' contributions}

Qiaolian Xu and Jun Wang designed the study, supervised the data collection, analyzed the data, interpreted the data, prepare the manuscript for publication and reviewed the draft of the manuscript. All authors have read and approved the manuscript.

\section{References}

1. Brun-Buisson C. The epidemiology of the systemic inflammatory response. Intensive Care Med. 2000; 26 Suppl 1: S64-S74, doi: 10.1007/s001340051121, indexed in Pubmed: 10786961.

2. Alberti C, Brun-Buisson C, Goodman SV, et al. European Sepsis Group. Influence of systemic inflammatory response syndrome and sepsis on outcome of critically ill infected patients. Am J Respir Crit Care Med. 2003; 168(1): 77-84, doi: 10.1164/rccm.200208-785OC, indexed in Pubmed: 12702548.

3. Juroš GF, Nikić MT, Šarić SD, Perić M, Rogić D. Contribution of presepsin, procalcitonin and C-reactive protein to the SOFA score in early sepsis diagnosis in emergency abdominal surgical patients. Signa Vitae. 2019; 15(1): 38, doi: 10.22514/ sv151.042019.5.

4. Qazi SA, Stoll BJ. Neonatal sepsis: a major global public health challenge. Pediatr Infect Dis J. 2009; 28(1 Suppl): S1-S2, doi: 10.1097/INF.0b013e31819587a9, indexed in $\mathrm{Pu}-$ bmed: 19106756.

5. Mayr FB, Yende S, Angus DC. Epidemiology of severe sepsis. Virulence. 2014; 5(1): 4-11, doi: 10.4161/viru.27372, indexed in Pubmed: 24335434.

6. Crouser ED. Mitochondrial dysfunction in septic shock and multiple organ dysfunction syndrome. Mitochondrion. 2004; 4(5-6): 729-741, doi: 10.1016/j.mito.2004.07.023, indexed in Pubmed: 16120428 .

7. Dickson RP, Singer BH, Newstead MW, et al. Enrichment of the lung microbiome with gut bacteria in sepsis and the acute respiratory distress syndrome. Nat Microbiol. 2016; 1(10): 16113, doi: $10.1038 /$ nmicrobiol.2016.113, indexed in Pubmed: 27670109.

8. Zhao H, Chen H, Xiaoyin M, et al. Autophagy activation improves lung injury and inflammation in sepsis. Inflammation. 2019; 42(2): 426-439, doi: 10.1007/s10753-018-00952-5, indexed in Pubmed: 30645707.

9. Sun LC, Zhang HB, Gu CD, et al. Protective effect of acacetin on sepsis-induced acute lung injury via its anti-inflammatory and antioxidative activity. Arch Pharm Res. 2018; 41(12): 1199-1210, doi: 10.1007/s12272-017-0991-1, indexed in Pubmed: 29243040.

10. Li L, Shu MQ, Chen J. CYLD deficiency exacerbates lipopolysaccharide (LPS)-induced pyroptosis in astrocytes of mice with sepsis. Biochem Biophys Res Commun. 2019; 514(4): 1066-1073, doi: 10.1016/j.bbrc.2019.05.033, indexed in $\mathrm{Pu}$ bmed: 31097224.

11. Wang P-L, Ohura K. Porphyromonas gingivalis lipopolysaccharide signaling in gingival fibroblasts-CD14 and Toll-like receptors. Critic Rev Oral Biol Med 2002; 13(2):132-142. , doi: 10.1177/154411130201300204, indexed in Pubmed: 12097356
12. Tse KH, Chow KBS, Leung WK, et al. Lipopolysaccharide differentially modulates expression of cytokines and cyclooxygenases in dorsal root ganglion cells via Toll-like receptor-4 dependent pathways. Neuroscience. 2014; 267: 241-251, doi: 10.1016/j.neuroscience.2014.02.041, indexed in Pubmed: 24607321.

13. Kurihara S, Hakuno F, Takahashi S. Insulin-like growth factor-I-dependent signal transduction pathways leading to the induction of cell growth and differentiation of human neuroblastoma cell line SH-SY5Y: the roles of MAP kinase pathway and PI 3-kinase pathway. Endocr J. 2000; 47(6): 739-751, doi: 10.1507/endocrj.47.739, indexed in Pubmed: 11228049.

14. Duan $\mathrm{C}, \mathrm{Xu}$ Q. Roles of insulin-like growth factor (IGF) binding proteins in regulating IGF actions. Gen Comp Endocrinol. 2005; 142(1-2): 44-52, doi: 10.1016/j.ygcen.2004.12.022, indexed in Pubmed: 15862547.

15. Lewitt MS, Boyd GW. The Role of Insulin-Like growth factors and insulin-like growth factor-binding proteins in the nervous system. Biochem Insights. 2019; 12: 1178626419842176, doi: 10.1177/1178626419842176, indexed in Pubmed: 31024217.

16. Jin Li, Shen F, Weinfeld M, et al. Insulin growth factor binding protein 7 (IGFBP7)-related cancer and IGFBP3 and IGFBP7 crosstalk. Front Oncol. 2020; 10: 727, doi: 10.3389/ fonc.2020.00727, indexed in Pubmed: 32500027.

17. Evdokimova V, Tognon CE, Benatar T, et al. IGFBP7 binds to the IGF-1 receptor and blocks its activation by insulin-like growth factors. Sci Signal. 2012; 5(255): ra92, doi: 10.1126/ scisignal.2003184, indexed in Pubmed: 23250396.

18. Tamura K, Hashimoto K, Suzuki K, et al. Insulin-like growth factor binding protein-7 (IGFBP7) blocks vascular endothelial cell growth factor (VEGF)-induced angiogenesis in human vascular endothelial cells. Eur J Pharmacol. 2009; 610(1-3): 61-67, doi: 10.1016/j.ejphar.2009.01.045, indexed in Pubmed: 19374835 .

19. Wajapeyee N, Serra RW, Zhu X, et al. Oncogenic BRAF induces senescence and apoptosis through pathways mediated by the secreted protein IGFBP7. Cell. 2008; 132(3): 363-374, doi: 10.1016/j.cell.2007.12.032, indexed in Pubmed: 18267069.

20. Abu-Safieh L, Abboud EB, Alkuraya H, et al. Mutation of IGFBP7 causes upregulation of BRAF/MEK/ERK pathway and familial retinal arterial macroaneurysms. Am J Hum Genet. 2011; 89(2): 313-319, doi: 10.1016/j.ajhg.2011.07.010, indexed in Pubmed: 21835307.

21. Benatar T, Yang W, Amemiya Y, et al. IGFBP7 reduces breast tumor growth by induction of senescence and apoptosis pathways. Breast Cancer Res Treat. 2012; 133(2): 563-573, doi: 10.1007/s10549-011-1816-4, indexed in Pubmed: 21997538.

22. Lee HSu, Kang P, Kim KaY, et al. Foeniculum vulgare Mill. Protects against Lipopolysaccharide-induced acute lung injury in mice through ERK-dependent NF- B activation. Korean J Physiol Pharmacol. 2015; 19(2): 183-189, doi: 10.4196/ kjpp.2015.19.2.183, indexed in Pubmed: 25729281.

23. de Oliveira MT, de Sá Coutinho D, Tenório de Souza É, et al. Orally delivered resveratrol-loaded lipid-core nanocapsules ameliorate LPS-induced acute lung injury via the ERK and PI3K/Akt pathways. Int J Nanomedicine. 2019; 14: 5215-5228, doi: 10.2147/IJN.S200666, indexed in Pubmed: 31371957.

24. Fei D, Meng X, Kang K, et al. Heme oxygenase-1 modulates thrombomodulin and activated protein $\mathrm{C}$ levels to attenuate lung injury in cecal ligation and puncture-induced acute lung injury mice. Exp Lung Res. 2012; 38(4): 173-182, doi: 10.3109/01902148.2012.660559, indexed in Pubmed: 22417130 . 
25. Jiang M, Qin C, Han M. Primary breast cancer induces pulmonary vascular hyperpermeability and promotes metastasis via the VEGF-PKC pathway. Mol Carcinog. 2016; 55(6): 1087 -1095, doi: 10.1002/mc.22352, indexed in Pubmed: 26152457.

26. Wang X, Ma T, Wan X, et al. IGFBP7 regulates sepsis induced acute kidney injury through ERK1/2 signaling. J Cell Biochem. 2018; 120(5): 7602-7611, doi: 10.1002/jcb.28035, indexed in Pubmed: 30450602.

27. Cunha BA. Sepsis and septic shock: selection of empiric antimicrobial therapy. Crit Care Clin. 2008; 24(2): 313-34, ix, doi: 10.1016/j.ccc.2007.12.015, indexed in Pubmed: 18361948.

28. Kelly-Scumpia KM, Scumpia PO, Weinstein JS, et al. B cells enhance early innate immune responses during bacterial sepsis. J Exp Med. 2011; 208(8): 1673-1682, doi: 10.1084/ jem.20101715, indexed in Pubmed: 21746813.

29. Chen Y, Liu Z, Pan T, et al. JMJD3 is involved in neutrophil membrane proteinase 3 overexpression during the hyperinflammatory response in early sepsis. Int Immunopharmacol. 2018; 59: 40-46, doi: 10.1016/j.intimp.2018.03.027, indexed in Pubmed: 29621735.

30. Miyashita T, Ahmed AK, Nakanuma S, Okamoto K, Sakai S, Kinoshita J, Makino I, Nakamura K, Hayashi H, Oyama K. A three-phase approach for the early identification of acute lung injury induced by severe sepsis. In Vivo. 2016; 30(4):341349. , indexed in Pubmed: 27381595.

31. Lelubre C, Vincent JL. Mechanisms and treatment of organ failure in sepsis. Nat Rev Nephrol. 2018; 14(7): 417-427, doi: 10.1038/s41581-018-0005-7, indexed in Pubmed: 29691495.

32. Thompson BT, Chambers RC, Liu KD. Acute Respiratory Distress Syndrome. N Engl J Med. 2017; 377(6): 562-572, doi: 10.1056/NEJMra1608077, indexed in Pubmed: 28792873.

33. Vijayan A, Faubel S, Askenazi DJ, et al. American Society of Nephrology Acute Kidney Injury Advisory Group. Clinical Use of the Urine Biomarker [TIMP-2] $\times$ [IGFBP7] for Acute Kidney Injury Risk Assessment. Am J Kidney Dis. 2016; 68(1): 19-28, doi: 10.1053/j.ajkd.2015.12.033, indexed in Pubmed: 26948834.

34. Vizioli MG, Sensi M, Miranda C, et al. IGFBP7: an oncosuppressor gene in thyroid carcinogenesis. Oncogene. 2010; 29(26): 3835-3844, doi: 10.1038/onc.2010.136, indexed in Pubmed: 20440262.

35. Schuh K, Pahl A. Inhibition of the MAP kinase ERK protects from lipopolysaccharide-induced lung injury. Biochem Pharmacol. 2009; 77(12): 1827-1834, doi: 10.1016/j. bcp.2009.03.012, indexed in Pubmed: 19428337.

Submitted: 1 September, 2020 Accepted after reviews: 13 December, 2020 Available as AoP: 16 December, 2020 